\title{
The outcome of severe varicella pneumonia with respiratory failure admitted to the intensive care unit for mechanical ventilation
}

To the Editor:

Varicella pneumonia carries a mortality of $10-30 \%$; mortality rate increases to $50 \%$ in patients that warrant mechanical ventilation secondary to respiratory failure despite appropriate antiviral and supportive treatment. Risk factors for the development of severe disease include cigarette smoking, pregnancy, immunocompromised states and more extensive skin involvement [1]. Treatment options for life-threatening varicella consist mainly of cardiorespiratory support, antiviral therapy and steroid use. The benefit of antiviral therapy, however, is still controversial [2].

Due to the paucity of data pertaining to varicella zoster pneumonia, attributed to its rarity, we aimed to describe all cases of varicella pneumonia admitted to an intensive care unit (ICU) that provides tertiary care to a population with a high HIV and tuberculosis burden. Moreover, we aimed to identify possible predictors of nonsurvival.

All cases of varicella pneumonia presenting to Tygerberg Academic Hospital adult respiratory intensive care unit between January 2004 and December 2016 were identified from an existing registry. Tygerberg Academic Hospital is a 1380-bed public hospital in South Africa serving 3 million people in and around the Cape Town metropole. The population served has a HIV prevalence of 5.2\% (95\% CI 3.4-7.8\%) [3, 4] and the incidence of tuberculosis was 100 cases per 100000 persons in 2016 [5]. Only patients aged $>18$ years with complete medical and radiological records were included in the final analysis. Ethical approval for the study was provided by the Stellenbosch University Research Ethics Committee (S16/04/082).

Apart from the general epidemiological characteristics, we specifically documented the presence or absence of comorbid diseases and other potential risk factors, including current or recent pregnancy, HIV infection, active or previous pulmonary tuberculosis, chronic obstructive pulmonary disease, obesity (body mass index $>30 \mathrm{~kg} \cdot \mathrm{m}^{-2}$ ), known malignancies and the use of immunosuppressive drugs.

The severity of illness was assessed by means of the Acute Physiology and Chronic Health Evaluation (APACHE) II score [6], which was calculated $24 \mathrm{~h}$ after admission to the ICU, as well as the arterial oxygen tension/inspiratory oxygen fraction ratio. We adapted a scoring system described by OpraviL et al. [7] to grade the severity and extent of pulmonary infiltrates: each lung was divided into four equal quadrants and each quadrant was scored on a scale of $0-3$ (0: normal; 1 : subtle increased interstitial markings; 2: prominent interstitial opacities; 3: confluent interstitial and acinar opacities), giving a maximum score of 24 for both lungs. Peripheral blood white cell counts and C-reactive protein (CRP) levels were performed on admission

We specifically noted the following complications defined according to accepted criteria: acute respiratory distress syndrome (ARDS) [8], septic shock [9], acute renal failure (ARF) [10] and ventilator-associated pneumonia (VAP) [11]. In the absence of a post mortem investigation, we accepted the opinion of the unit's morbidity and mortality committee regarding the cause of death.

@ERSpublications

Varicella pneumonia still carries a high mortality rate $(29.2 \%$ in this study). Metabolic acidosis, a higher APACHE II score and the presence of acute renal failure on admission predicted poor outcome. http://ow.ly/CdQM30jAY5q

Cite this article as: John KG, John T-J, Taljaard JJ, et al. The outcome of severe varicella pneumonia with respiratory failure admitted to the intensive care unit for mechanical ventilation. Eur Respir J 2018; 52: 1800407 [https://doi.org/10.1183/13993003.00407-2018]. 
IBM SPSS version 24 (IBM Corp, Armonk, NY, USA) was used to analyse the data. Due to the small sample size, only bivariate analysis was carried out to examine associations between risk factors and mortality. For continuous variables, nonparametric Mann-Whitney tests were used, and for categorical variables, Fisher's exact tests were used. Where possible, odds ratios (95\% CI) were calculated for dichotomous risk factors that were found to have a p-value $<0.05$. Unless stated otherwise, data are presented as median (interquartile range).

A total of 26 cases of severe varicella pneumonia requiring ventilatory support were identified during the study period. Two were excluded from further analysis due to incomplete medical and radiological records. Of the 24 patients included (age 30.0 (27.0-33.5) years), 15(62.5\%) were females. Notable risk factors included HIV coinfection $(\mathrm{n}=10,41.7 \%)$, pregnancy/puerperium $(\mathrm{n}=8,33.3 \%)$ and underlying tuberculosis $(\mathrm{n}=7,29.2 \%)$. The mean duration from initial onset of symptoms to ICU admission was $3.9 \pm 1.5$ days (range 1-7 days). Seven patients died (ICU mortality 29.2\%). No risk was associated with nonsurvival. All patients were treated with intravenous acyclovir $\left(5 \mathrm{mg} \cdot \mathrm{kg}^{-1}\right.$ 8-hourly for 10 days $)$ and 20 were treated with intravenous hydrocortisone. Neither the addition of hydrocortisone to acyclovir (mortality six out of 20 versus one out of four, $\mathrm{p}=1)$ nor the dose thereof, which varied from $50 \mathrm{mg}(\mathrm{n}=6)$ to $100 \mathrm{mg}(\mathrm{n}=12)$ to $200 \mathrm{mg}(\mathrm{n}=2)$, had an impact on survival.

A bilateral nodular appearance on the chest radiograph was the most common radiological finding $(n=22$, 91.7\%), although no specific radiological pattern or radiological score had a significant effect on prognosis. The radiological score for survivors was 19.0 (180-22.0) and nonsurvivors $20.0(17.0-22.0)(\mathrm{p}=0.42)$. The indices of disease severity and common complications are summarised in table 1. Metabolic acidosis ( $\mathrm{pH} 7.11$ versus 7.39, $\mathrm{p}=0.020 ; \mathrm{HCO}_{3}^{-} 14.3$ versus $24.5 \mathrm{mEq} \cdot \mathrm{L}^{-1}, \mathrm{p}=0.016$ ) and a higher APACHE II score ( 22.0 versus $13.0, \mathrm{p}=0.009$ ) were significantly associated with nonsurvival. ARF on admission $(\mathrm{p}=0.023)$ was found to be a risk factor for mortality, with an odds ratio of 14.4 (95\% CI 1.4-152.5).

Other complications (ARDS, septic shock, secondary infections and VAP) were equally distributed between the groups.

In our study population, which to the best of our knowledge, included the largest number of cases from a single-centre cohort, we found that varicella pneumonia necessitating ICU admission had a mortality of $\sim 30 \%$. Disease severity indicators (metabolic acidosis $(\mathrm{p}=0.02)$, a higher APACHE II score and ARF on admission) were significant predictors of mortality, whereas risk factors, chest radiography and secondary infections were not.

TABLE 1 Disease severity and complications related to varicella pneumonia on admission

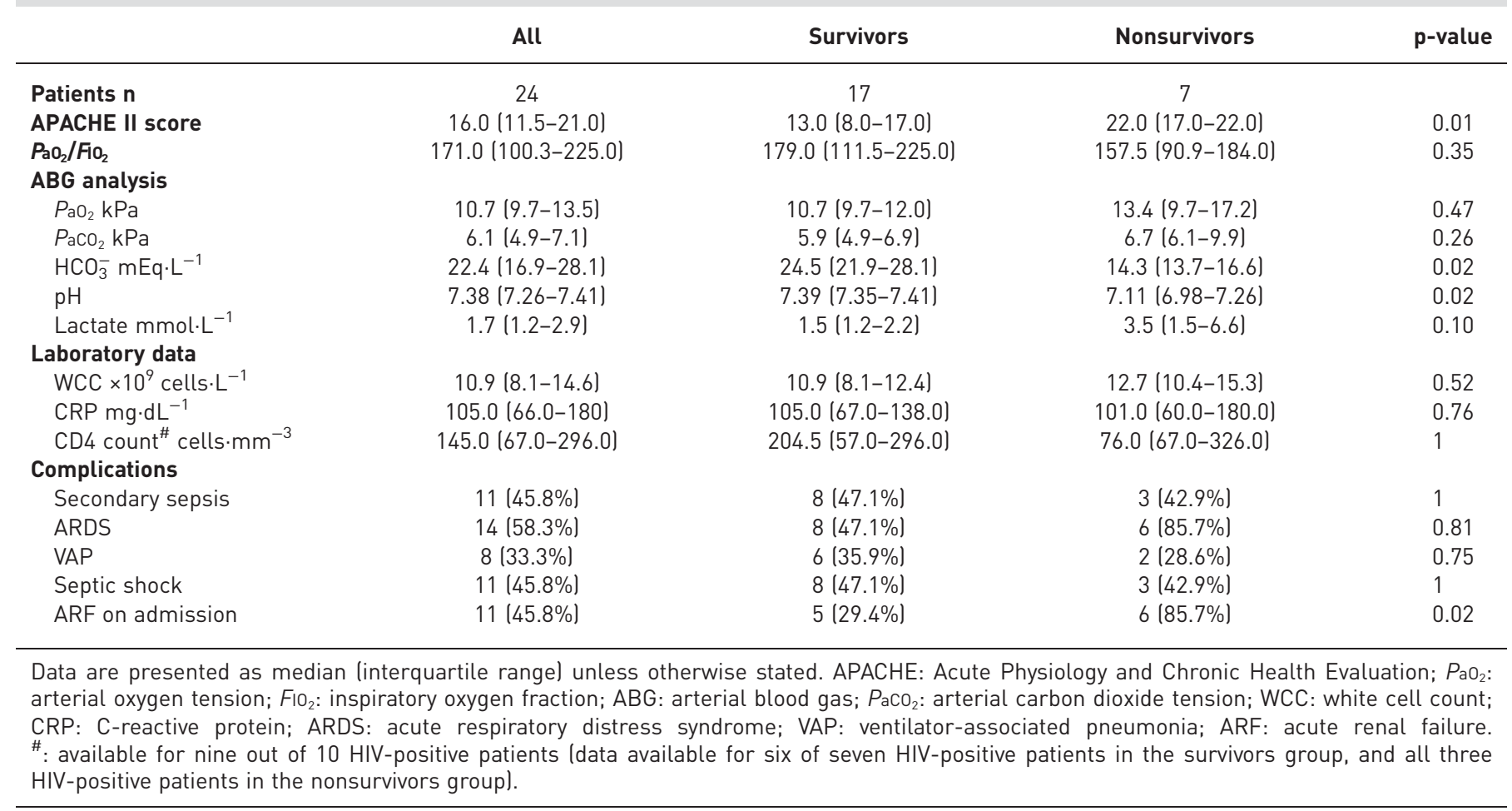


General risk factors for varicella pneumonia in the adult population include cigarette smoking, pregnancy, immunocompromised states and a greater number of skin lesions [12]. Despite the fact that all our patients had at least one or more risk factor, none were found to predict nonsurvival. These findings are comparable to previous studies suggesting that the underlying medical context was no longer significantly associated with prognosis after adjustment for the severity of the acute disease [13].

Treatment options for life-threatening varicella include cardiorespiratory support, antiviral therapy and corticosteroids. In a retrospective study of 99 patients, no benefit was shown prior to and after the introduction of acyclovir [2]. Despite its limited efficacy, it is still widely recommended as an early primary therapy [14, 15]. Earlier studies (all with small sample sizes) suggested a mortality benefit, reduced ICU stay and even more rapid radiological improvement [2]. A large, recent French multicentre retrospective trial failed to confirm these potential benefits. In fact, steroid use was associated with more ICU-acquired infections [13]. The present study also could not demonstrate any survival benefit from the addition of steroids to acyclovir.

A recent study showed that severity of respiratory disease, early bacterial co-infection and other organ failure on ICU admission were independent risk factors for invasive mechanical ventilation but due to the limited number of deaths, they were unable to identify predictors of hospital mortality [13]. Apart from acute renal failure, our data failed to show an association between early bacterial co-infection and other organ failure on ICU admission and mortality.

Severe varicella is fortunately relatively rare, which makes large prospective studies very difficult. The major strengths of our study are the relative large sample size and the fact that data were missing in only two out of 26 patients. Potential limitations include the retrospective nature and the fact that the absolute numbers may compromise efforts to obtain statistical significance. Moreover, it should be noted that ARF, acidosis and the APACHE II score are all related, and not independent parameters, which combined with the sample size, precluded a multivariate analysis. Other limitations include the single-centre design and the fact that the data collection spanned 13 years, during which evidence-based ICU management constantly evolved.

In conclusion, we found that varicella pneumonia in our population still carries a high mortality rate (29.2\%). Metabolic acidosis, a higher APACHE II score and the presence of ARF on admission predicted poor outcome. Further prospective, multicentre studies with larger cohorts may be needed to identify other prognostic factors.

Kiran George John ${ }^{1}$, Thadathilankal-Jess John ${ }^{1}$, Jantjie J. Taljaard ${ }^{2}$, Usha Lalla ${ }^{1}$, Tonya M. Esterhuizen ${ }^{3}$, Elvis M. Irusen ${ }^{1}$ and Coenraad F.N. Koegelenberg ${ }^{1}$

${ }^{1}$ Division of Pulmonology, Dept of Medicine, Stellenbosch University and Tygerberg Academic Hospital, Cape Town, South Africa. ${ }^{2}$ Division of Infectious Diseases, Dept of Medicine, Stellenbosch University and Tygerberg Academic Hospital, Cape Town, South Africa. ${ }^{3}$ Division of Epidemiology and Biostatistics, Dept of Global Health, Stellenbosch University and Tygerberg Academic Hospital, Cape Town, South Africa.

Correspondence: Kiran George John, Division of Pulmonology, Dept of Medicine, Stellenbosch University and Tygerberg Academic Hospital, PO Box 241, Cape Town, 8000, South Africa. E-mail: kirangeorge23@gmail.com

Received: Feb 142018 | Accepted after revision: April 042018

Conflict of interest: None declared.

\section{References}

Mohsen AH, McKendrick M. Varicella pneumonia in adults. Eur Respir J 2003; 21: 886-891.

Mer M, Richards GA. Corticosteroids in life-threatening varicella pneumonia. Chest 1998; 114: 426-431.

John T-J, Plekker D, Irusen EM, et al. Does access to private healthcare influence potential lung cancer cure rates? South African Med J 2017; 107: 687.

4 Shisana O, Rehle T, Simbayi L. South African National HIV Prevalence, Incidence and Behaviour Survey. South African National HIV Prevalence, Incidence and Behaviour Survey. www.hsrc.ac.za/en/research-data/view/6871 Date last updated: 2014. Date last accessed: December 8, 2015.

5 World Health Organization. Global tuberculosis control. Global tuberculosis control Report 2017. www.who.int/tb/ publications/global_report/en/ Date last updated: 2017. Date last accessed: January 4, 2018.

6 Knaus WA, Draper EA, Wagner DP, et al. APACHE II: a severity of disease classification system. Crit Care Med 1985; 13: 818-829.

7 Opravil M, Marincek B, Fuchs WA, et al. Shortcomings of chest radiography in detecting Pneumocystis carinii pneumonia. J Acquir Immune Defic Syndr 1994; 7: 39-45.

8 Ranieri VM, Rubenfeld GD, Thompson BT, et al. Acute respiratory distress syndrome: the Berlin Definition. JAMA 2012; 307: 2526-2533.

9 Rhodes A, Evans LE, Alhazzani W, et al. Surviving Sepsis Campaign: international guidelines for management of sepsis and septic shock: 2016. Crit Care Med 2017; 45: 486-552.

10 Kellum JA, Lameire N, Aspelin P, et al. Kidney Disease: Improving Global Outcomes (KDIGO) Acute Kidney Injury Work Group. KDIGO Clinical Practice Guideline for Acute Kidney Injury. Kidney Int 2012; 2: 1.

11 Torres A, Ewig S. Diagnosing ventilator-associated pneumonia. N Engl J Med 2014; 350: 433-435. 
12 Baren JM, Henneman PL, Lewis RJ. Primary varicella in adults: pneumonia, pregnancy, and hospital admission. Ann Emerg Med 1996; 28: 165-169.

13 Mirouse A, Vignon P, Piron P, et al. Severe varicella-zoster virus pneumonia: a multicenter cohort study. Crit Care 2017; 21: 137.

14 Haake DA, Zakowski PC, Haake DL, et al. Early treatment with acyclovir for varicella pneumonia in otherwise healthy adults: retrospective controlled study and review. Rev Infect Dis 1990; 12: 788-798.

15 Davidson RN, Lynn W, Savage P, et al. Chickenpox pneumonia: experience with antiviral treatment. Thorax 1988; 43: 627-630. 